\title{
Critical Parameters of Solution Design for Electrodeposition of 2.4 T CoFe Alloys
}

\author{
S. R. Brankovic ${ }^{\mathrm{a}, \mathrm{b}, \mathrm{c}}$, J. George ${ }^{\mathrm{a}}$, S. -E. Bae ${ }^{\mathrm{a}}$ and D. Litvinov ${ }^{\mathrm{a}, \mathrm{c}}$ \\ ${ }^{a}$ Electrical and Computer Eng. Dep., University of Houston, TX 77204-4005, USA \\ ${ }^{b}$ Chemical and Bimolecular Eng. Dep., University of Houston, TX 77204-4004, USA \\ ${ }^{c}$ Center for Nanomagnetic Systems, University of Houston, TX 77204-4005, USA
}

\begin{abstract}
The electrodeposition of soft, high magnetic moment alloys has become the critical fabrication step in manufacturing of magnetic recording heads. The need for ultimately high magnetic moment alloys $(2.4 \mathrm{~T}$ CoFe $)$ and the electrodeposition process capable of delivering magnetic structures with dimension in the range of several tens of nanometers are the new tasks that have to be addressed if the future magnetic recording devices will use the electrodeposited alloys for writing pole application. This complex challenge could be successfully achieved by fully considering the conditions at the electrochemical interface for additive adsorption and incorporation, iron hydroxide formation and incorporation, and by careful design of the bath chemistry and deposition parameters that would yield the high moment alloys with the optimum magnetic properties. In this manuscript, some of the key aspects of successful electrodeposition of $2.4 \mathrm{~T}$ CoFe alloys are discussed with experimental results and theoretical foundations indicating future direction for development of electrodeposited high moment alloys and nanostructures.
\end{abstract}

\section{Introduction}

The introduction of the perpendicular magnetic recording heads design (1) and magnetic media with significantly larger anisotropy and coercivities have posted the need for electrodeposited $\mathrm{CoFe}$ alloys with the highest magnetic moment possible. Being the one of the crucial steps in fabrication of magnetic recording heads, the electrodeposition of CoFe alloys is been used to create the magnetic structures with sub-100 nm critical dimensions. These challenges require that the electrodeposition parameters determining the properties of the CoFe alloys at nanoscale are examined in more details in order to define electrodeposition process for nanostructures with optimum properties and performance. 


\section{Experimental}

A standard three electrode cell configuration with solution volume of $5 \mathrm{~L}$ was used to electrodeposit $0.6 \pm 0.05 \mu \mathrm{m}$ thick $\mathrm{Co}_{40-45} \mathrm{Fe}_{60-55}$ films. The cathodes were rotating $\mathrm{Cu}$ disks $(\mathrm{D}=1.7 \mathrm{~cm})$ electropolished to a mirror finish and a Co plate was used as an anode. The electrodeposition is carried out under galvanostatic control and solution design and parameters of electrodeposition process are described in Table I. The additive used in the deposition experiments was saccharin with concentration ranging from 0 . $\mathrm{g} \cdot \mathrm{L}^{-1}$ to $2.5 \mathrm{~g} \cdot \mathrm{L}^{-1}$. The composition of electrodeposited films was verified by Energy Dispersive X-ray Spectroscopy (EDX). The magnetic properties of the CoFe films were verified using Vibrating Sample Magnetometer (VSM). The thickness of the $\mathrm{CoFe}$ films was determined from Scanning Electron Microscopy (SEM) images of focused ion beam (FIB) cross-sections and from charge stripping measurements. Upon the deposition of CoFe films and verification of their magnetic properties, thickness and composition, the samples were subjected to Wavelength Dispersive X-ray Spectroscopy (WDS) analysis to determine the amount of

\begin{tabular}{|c|c|c|c|}
\hline \multicolumn{2}{|c|}{ 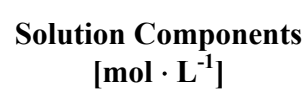 } & \multicolumn{2}{|c|}{ Process Parameters } \\
\hline $\mathrm{FeSO}_{4}$ & 0.1 & $j$ & $3.8-4.0 \mathrm{~mA} \cdot \mathrm{cm}^{-2}$ \\
\hline $\mathrm{CoSO}_{4}$ & 0.046 & $\omega$ & $300 \mathrm{rpm}$ \\
\hline$\left(\mathrm{NH}_{4}\right)_{2} \mathrm{SO}_{4}$ & 0.3 & $\gamma$ & $\sim 0.12$ \\
\hline $\mathrm{H}_{3} \mathrm{BO}_{3}$ & 0.4 & $p H$ & 2.0 \\
\hline$T$ & $298 \mathrm{~K}$ & $D_{H+} \delta$ & $0.015 \mathrm{~cm} \cdot \mathrm{s}^{-1}$ \\
\hline \multicolumn{4}{|c|}{$\begin{array}{l}{\left[\mathrm{Fe}^{3+}\right]_{i}^{*} \approx 0.00128 \mathrm{~mol} \cdot \mathrm{L}^{-1}} \\
{\left[\mathrm{Fe}^{3+}\right]^{*} \approx 0.0028 \mathrm{~mol} \cdot \mathrm{L}^{-1}}\end{array}$} \\
\hline
\end{tabular}
incorporated sulfur in the deposit.

\section{Relation Between Surface Concentration of $\mathrm{H}^{+}$and Concentration of $\mathrm{Fe}^{3+}$ in the Bulk Solution - The Useful Criteria for Process Design and Control.}

\subsection{Direct Current Deposition.}

The depletion of the hydrogen ions at the electrode/solution interface during electrodeposition of magnetic alloys leads to the local increase in $p H$. This effect promotes the formation of insoluble hydroxide species at the electrode/solution interface and it is attributed as the main reason for incorporation of oxygen and nonmagnetic inclusions in $2.4 \mathrm{~T}$ CoFe alloys $(2,3)$. The incorporation of metal hydroxides into deposit inevitably results in dilution of the saturation magnetic flux density of electrodeposited films. The most insoluble metal hydroxide when $\mathrm{Co}$ and $\mathrm{Fe}$ are considered is $\mathrm{Fe}(\mathrm{OH})_{3}$ with the product of solubility, $K_{p}=2.79 \times 10^{-39} \mathrm{~mol}^{4} \cdot \mathrm{L}^{-4}$, (4). The presence of $\mathrm{Fe}^{3+}$ and 
$\mathrm{Fe}(\mathrm{OH})_{3}$ in the plating solutions is mainly due to dissolved oxygen from air which oxidizes $\mathrm{Fe}^{2+}$ ions into $\mathrm{Fe}^{3+}$. The necessary condition for $\mathrm{Fe}(\mathrm{OH})_{3}$ precipitation from the bulk solution for known $\mathrm{pH}$ of solution could be expressed in terms of the bulk $\mathrm{Fe}^{3+}$ concentration $\left(\left[\mathrm{Fe}^{3+}\right]_{\infty}\right)$ as, (5):

$$
\left[F e^{3+}\right]_{\infty}^{*}=\frac{K_{p}}{\left(K_{w}\right)^{3}} \cdot 10^{-3 \cdot p H}
$$

Here, $K_{w}$ is the ionic product of water, and $K_{p}$ is defined previously. If the net flux of hydrogen ions towards the electrode surface through the diffusion layer during the galvanostatic deposition is expressed in terms of current density $j$ and current efficiency $\gamma$ as; $j(1-\gamma) / F$, then the concentration of the hydrogen ions at the electrode/solution interface, $\left[\mathrm{H}^{+}\right]_{i}$, could be estimated from the simple mass balance described by the Fick's first law of diffusion (6);

$$
\left[H^{+}\right]_{i}=\left(10^{-p H}-\frac{(1-\gamma) \cdot j}{F} \cdot \frac{\delta}{D_{H^{+}}}\right)
$$

In above expression, $F$ is Faraday's constant, $\delta$ is the diffusion layer thickness and $D_{H}{ }^{+}$ is diffusivity of $\mathrm{H}^{+}$. Combining equations [2] and [3], the concentration of the $\mathrm{Fe}^{3+}$ at the electrode/solution interface, $\left[\mathrm{Fe}^{3+}\right]_{i}^{*}$ above which the precipitation of $\mathrm{Fe}(\mathrm{OH})_{3}$ starts is expressed as:

$$
\left[F e^{3+}\right]_{i}^{*}=\frac{K_{p}}{\left(K_{w}\right)^{3}} \cdot\left(10^{-p H}-\frac{(1-\gamma) \cdot j}{F} \cdot \frac{\delta}{D_{H^{+}}}\right)^{3}
$$

In order to illustrate the effect of $\mathrm{Fe}^{3+}$ ions presence in the plating solution on the magnetic moment of electrodeposited $2.4 \mathrm{~T}$ CoFe films the $B_{s}$ measurements as a function of the $\mathrm{Fe}^{3+}$ concentration in the plating solution are shown in Figure 1. The other parameters of the electrodeposition process are the described in Table I. As one can see, the magnetic moment of CoFe films starts to decrease for $\mathrm{Fe}^{3+}$ concentration that is very close to the calculated value of $\left[\mathrm{Fe}^{3+}\right]_{i}^{*}$ which indicates the direct correlation between the decrease of $B_{s}$ and the onset of $\mathrm{Fe}(\mathrm{OH})_{3}$ precipitation at the electrode/solution interface (6). These data also show that the increase of $\mathrm{Fe}^{3+}$ concentration beyond the calculated value of $\left[\mathrm{Fe}^{3+}\right]_{i}^{*}$ results in reduction of $\mathrm{B}_{\mathrm{s}}$ of $\mathrm{CoFe}$ films for $\sim 40 \%$ as compared to $B_{S}$ of corresponding bulk CoFe alloys. 

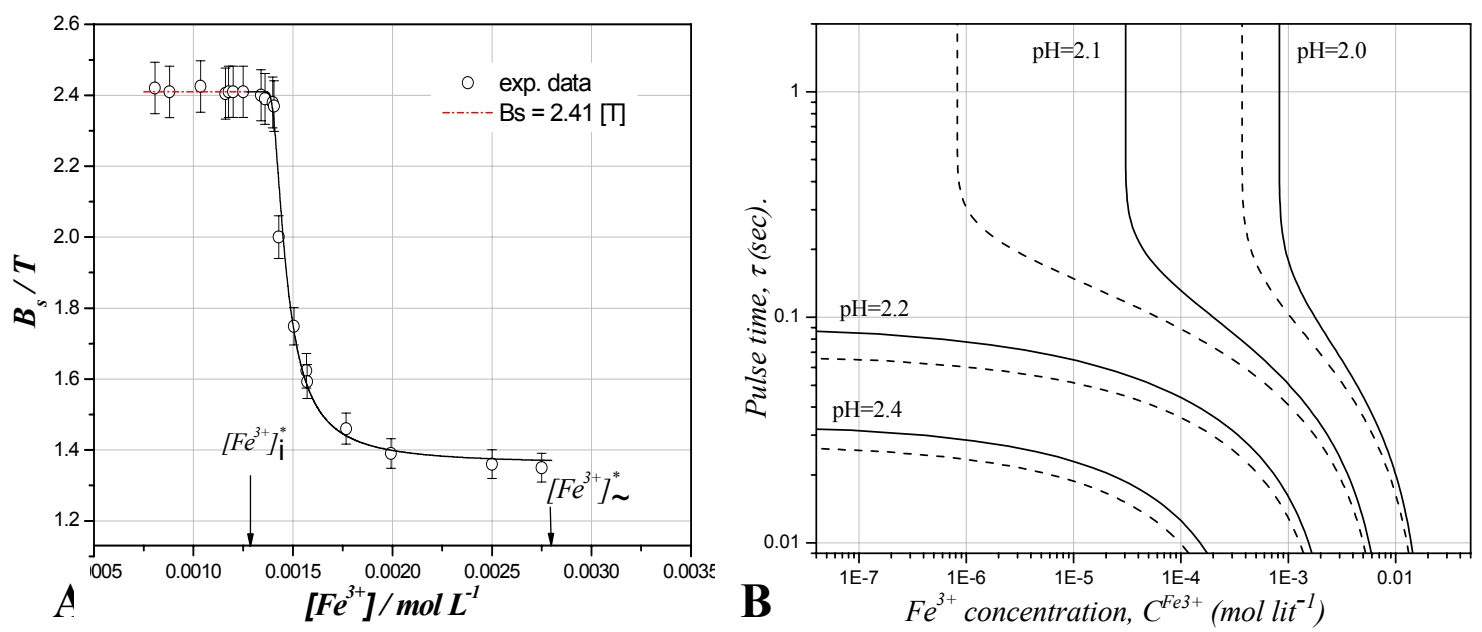

Figure 1. (A) Bs. vs. $\left[\mathrm{Fe}^{3+}\right]$ dependence for electrodeposited $\mathrm{CoFe}$ alloys. Parameters of deposition process and solution design are given in Table I. Saccharin concentration in the bath is $0.12 \mathrm{~g} \cdot \mathrm{L}^{-1}$. (B) The maximum duration of the pulse stage, $\tau$, as a function of the $\left[\mathrm{Fe}^{3+}\right]$. The dependence is presented for four different $p H$ values of the solution and two pulse current densities, $20 \mathrm{~mA} \cdot \mathrm{cm}^{-2}$ (solid line) and $22 \mathrm{~mA} \cdot \mathrm{cm}^{-2}$ (dashed line). The parameters of the solution and saccharin concentration are same as in (A).

Considering the expressions defining the values of $\left[\mathrm{H}^{+}\right]_{i}$ and $\left[\mathrm{Fe}^{3+}\right]_{i}^{*}$ (equations $[1$ $3]$ ) it is quite obvious that optimum condition for direct current deposition of $2.4 \mathrm{~T} \mathrm{CoFe}$ films are very sensitive to small changes in $p H$ or magnitude of the deposition current density. In general, for direct current deposition process of any soft magnetic alloys, the bath control should be such that allowed $\mathrm{Fe}^{3+}$ concentration in the solution is always significantly smaller than the calculated value of $\left[\mathrm{Fe}^{3+}\right]_{i}^{*}$. This fact hast to be taken with great attention if the direct current deposition process and bath design $(p H, \gamma, j)$ is intended to produce the magnetic films and nanostructures with $B_{s}$ values comparable to the bulk metallurgical alloys of the same composition.

\section{$\underline{3.2 \text { Pulse Current Deposition. }}$}

The typical pulse current function that is employed in electrodeposition process of soft magnetic alloys for magnetic recording application has the simple on/off profile $(5,7,8)$. The successful application of this pulse function is dependent on the proper determination of the pulse time $t_{\text {on }}$, and the magnitude of the pulse current density $j_{\text {pulse }}$. The useful approach to design pulse current function is based on the criteria that ensures stable electrode/solution interface with respect to $\mathrm{Fe}(\mathrm{OH})_{3}$ precipitation.

The optimum duration of the pulse current stage should be such that no limitations for $\mathrm{H}^{+}$transport towards the electrode-electrolyte interface is encountered. The mathematical treatment of the ion diffusion problem through the Nernst diffusion layer during the galvanostatic pulse means the solving Fick's second law of diffusion with 
appropriate initial and boundary conditions (9). The exact mathematical solution treatment of this problem is somewhat cumbersome, presented in the form of a Fourier series expansion (9). For practical application, it is more convenient to consider approximate solutions;

$$
\left[H^{+}\right]_{i}=\left[H^{+}\right]_{\infty}-\frac{(1-\gamma) \cdot j_{\text {pulse }} \cdot \delta}{F \cdot D^{H^{+}}} \cdot\left\{1-\frac{8}{\pi^{2}} \cdot \exp \left(-\frac{\pi^{2} \cdot D^{H^{+}}}{4 \delta^{2}} \cdot t\right)\right\}
$$

By performing the same exercise as in the previous discussion (equations (1)-(3)) the equation [4] can be used to define the concentration of the $\mathrm{Fe}^{3+}$ at the electrode solution interface for which the precipitation of hydroxide starts as a function of the pulse current density, $j_{\text {pulse }}$, pulse time, $t$, and diffusion layer thickness, $\delta$. However, the more useful exercise is to use the above equation for the estimate of the maximum duration of the pulse stage, $\tau$, which is defined by formation of the conditions allowing the precipitation of $\mathrm{Fe}(\mathrm{OH})_{3}$ at the interface which is presented as (5);

$$
\tau=-\frac{4 \delta^{2}}{\pi^{2} D_{H^{+}}} \cdot \ln \left\{\frac{\pi^{2}}{8} \cdot\left(1-\frac{F \cdot D_{H^{+}} \cdot\left[10^{-p H}-\sqrt[3]{\frac{K_{w}^{3}}{K_{p}} \cdot\left[F e^{3+}\right]_{\infty}}\right]}{(1-\gamma) \cdot j_{p u l s e} \cdot \delta}\right)\right\}
$$

The predictions of equation [5] are plotted in Figure 1B for different $p H$ values of the solution and two different pulse current densities. The pulse time duration estimate is presented only for the values larger than $0.01 \mathrm{~s}$ because for shorter pulse times the accuracy of the equation [4] is not satisfactory. For the design of the pulse time interval $t_{o n}$, the general rule should be to have the length of the pulse shorter than the maximum one estimated by equation [5], $t_{\text {on }}<\tau$.

From Figure $1 \mathrm{~B}$ it is obvious that a small variation in $p H$ of the solution can significantly affect the allowed duration of the pulse stage. For example, for a $10^{-3} \mathrm{~mol} \cdot \mathrm{L}^{-}$ ${ }^{1}$ concentration of $\mathrm{Fe}^{3+}$, and pulse current density of $20 \mathrm{~mA} \cdot \mathrm{cm}^{-2}$ the increase in the $\mathrm{pH}$ of the solution for $\sim 5 \%$ (from 2 to 2.1 ) leads to a decrease of the $\tau$ for more than $300 \%$. Knowing that, in the real situation, a $5 \%$ variation in $p H$ is considered very possible scenario, one can anticipate the significant restriction of $t_{o n}$ as compared to the estimated value of $\tau$ for a given concentration of $\mathrm{Fe}^{3+}$ in the solution. An additional way of interpreting these calculations is to consider the variation of $\tau$ with an increasing pulse current density. For the same concentration of $\mathrm{Fe}^{3+}$, but for an increase of $10 \%$ in $j_{\text {pulse }}$, the value of $\tau$ decreases by $\sim 40 \%$. This is important when considering the geometrical and secondary current distribution effects (10) on through-mask electrodeposition process of nanoscale features. A significant variation of the local current density $j_{\text {local }}$ within the photoresist pattern defining the magnetic pole electrode geometry could add an additional constraint on the estimate of $t_{o n}$ as compared to the ones shown in Figure 1B. 


\section{Additive Design}

In order to obtain magnetic alloys with lower coercivity, different additives have been used in the plating solutions $(11,12,13,14,15)$. In addition to producing the soft alloys, the common action of additives is expressed through the leveling and brightening of the deposit, improvement of the crystal structure $(13,16$,$) and the reduction of the$ residual stresses (17). The last two effects are also important for obtaining ultimately soft CoFe alloys. The use of additives in the plating solutions results in incorporation of different amounts of nonmagnetic interstitials and inclusions in the magnetic deposit (8, 16,18). Entire additive molecules or molecular fragments can be found in deposit as well $(19,20,21)$. If the amount of the incorporated additives is small, it is generally considered as beneficial for the desired magnetic softness (13). However, the significant presence of sulfur, metal sulfides or S-containing organic molecules and fragments, can cause deterioration of the magnetic alloy corrosion resistance $(22,23)$. For these reasons, the benefit and disadvantage of additive incorporation for properties of $\mathrm{CoFe}$ alloys has to be considered with great attention.

\section{$\underline{4.1 \text { Saccharin Adsorption }}$}

The most common additive used for electrodeposition of soft magnetic alloys for magnetic recoding application is saccharine and its derivatives. The saccharin represents the condensed organic molecule having benzyl and heterocyclic rings in its structure. During electrodeposition process, saccharin adsorbs on the metal surface forming some type of condensed phase (film) $(8,24)$. The density and the coverage of the adsorbed saccharine phase are dependent on the potential of electrode surface and the concentration of this additive in the plating solution (25). Useful information about saccharine adsorption and coverage is deduced from the impedance spectroscopy measurements where the potential vs. apparent saccharin coverage for particular electrode surface is determined using following expression (25);

$$
\theta=\frac{C_{\max }-C}{C_{\max }-C_{\min }}
$$

Here, $\mathrm{C}_{\max }, \mathrm{C}_{\min }$ and $\mathrm{C}$ are determined from impedance measurements and they represents the maximum and minimum value of the double layer capacitance for the potential range of capacitance well and the capacitance of the metal surface related to the potential within the capacitance well. The examples of this measurement are shown in Figure 2 for $2.4 \mathrm{~T}$ CoFe alloy surfaces in solutions with different content of saccharine. The apparent maximum coverage of saccharine for CoFe surfaces is around $-1.3 \mathrm{~V}$, Figure 2 . 


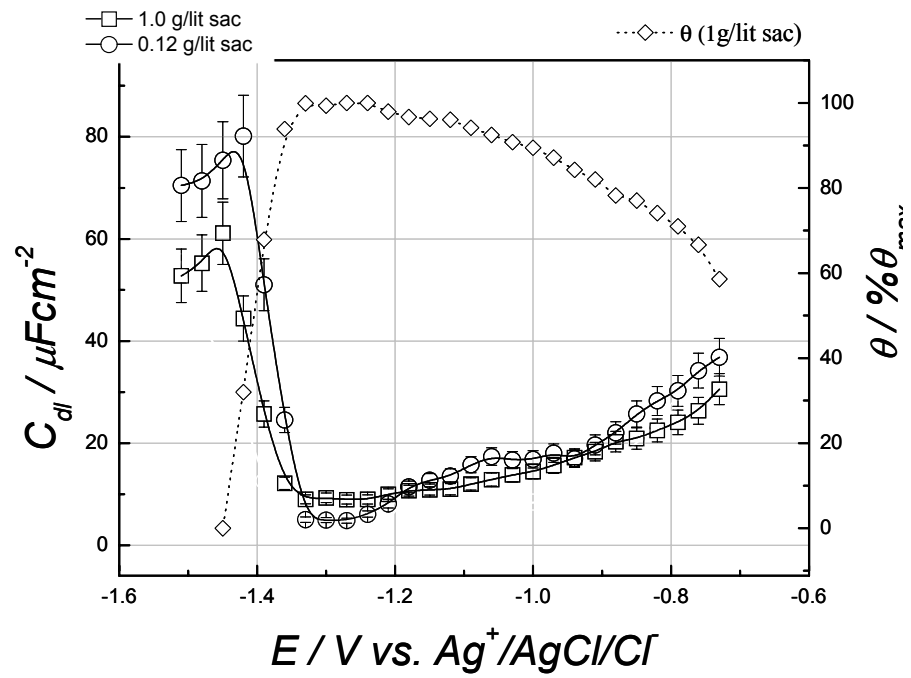

Figure 2. The double layer capacitance vs. potential dependence extracted from the impedance measurements for $2.4 \mathrm{~T} \mathrm{CoFe}$ alloy surface in solutions with different saccharin content. The corresponding coverage of saccharine is calculated using equation [6] and shown on the right ordinate. Solution details are presented in Table I.

\subsection{Saccharine as a Source of Sulfur in the Deposit.}

The sulfur incorporation into magnetic deposit coming from the saccharin as an additive occurs through the saccharin adsorption-electroreduction process or via physical incorporation of entire saccharin molecules during the deposit growth (21). The first mechanism represents the chemical route responsible for incorporating S-containing molecular fragments and metal sulfides in the deposit, while the second one represents the incorporation of entire molecules of saccharin having the sulfur atom as their integral part. In addition to these two additive related mechanisms, the physical entrapment of sulfur containing ions $\left(\mathrm{SO}_{4}{ }^{2-}, \mathrm{SO}_{3}{ }^{2-} \ldots\right)$ contributes to the sulfur content in the magnetic deposit as well. The overall sulfur incorporation rate $R_{S}\left[\mathrm{~mol} \cdot \mathrm{cm}^{-2} \mathrm{~s}^{-1}\right]$ into magnetic alloy can be described as the sum of the contributions coming from each of these mechanisms (21);

$$
R_{S}=R_{0}+K_{1} \cdot \theta+K_{2} \cdot \theta \cdot(1-\theta)
$$

In the above expression, the first term $R_{0}\left[\mathrm{~mol} \cdot \mathrm{cm}^{-2} \mathrm{~s}^{-1}\right]$ represents the sulfur incorporation as a part of trapped sulfates or other anions in the deposit which is independent on saccharine, the $K_{l} \cdot \theta$ term $\left[\mathrm{mol} \cdot \mathrm{cm}^{-2} \mathrm{~s}^{-1}\right]$ represent the sulfur incorporation via electroreduction of saccharine molecules (sulfur is incorporated as a part of metal sulfides) and the last term, $K_{2} \cdot \theta \cdot(1-\theta)\left[\mathrm{mol} \cdot \mathrm{cm}^{-2} \mathrm{~s}^{-1}\right]$ represents the sulfur incorporation as a part of the trapped saccharine molecules during the deposit growth (21). The $K_{1}$ and $K_{2}$ represent the saccharin electroreduction and incorporation rate constants expressed in $\left[\mathrm{mol} \cdot \mathrm{cm}^{-2} \mathrm{~s}^{-1}\right]$ units. If the saccharin adsorption is described in terms of Langmuir formalism (25), the more practical form of the model describing the sulfur incorporation rate is presented in terms of the saccharin concentration in the solution, $C_{s a c},(21)$; 


$$
R_{S}=R_{0}+K_{1} \cdot \frac{b \cdot C_{s a c}}{1+b \cdot C_{s a c}}+K_{2} \cdot \frac{b \cdot C_{s a c}}{\left(1+b \cdot C_{s a c}\right)^{2}}
$$

Here, $b$ is the saccharin adsorption constant, while the other terms are described previously. The example of sulfur incorporation rate into $2.4 \mathrm{~T}$ CoFe alloys as a function of saccharine concentration in the plating solution is shown in Figure 3. The dash-dot line in the same figure represents the model fit (equation [8]) to the experimental data. As it could be seen, the model succeeds to qualitatively explain experimental results.

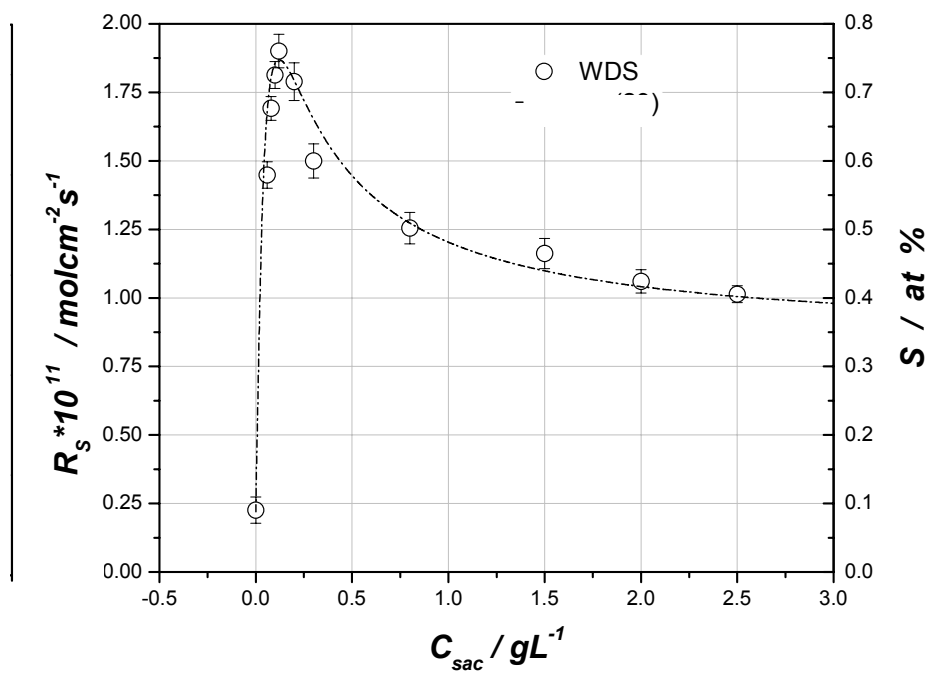

Figure 3. $R_{S}$ vs. $C_{s a c}$ dependence for direct current deposition of $2.4 \mathrm{~T}$ CoFe alloys. On the right ordinate the corresponding at $\%$ of $S$ in the deposit is indicated. Solution formulation and process parameters are the same as in Table I.

The mathematical nature of equation [8] allows performing a deconvolution of the sulfur incorporation rate on separate contributions of each incorporation mechanism. Also, it is straight forward to show that for $\boldsymbol{C}_{s a c}<\frac{\boldsymbol{K}_{2}-\boldsymbol{K}_{1}}{\boldsymbol{K}_{1} \cdot \boldsymbol{b}}$ the sulfur incorporation into magnetic deposit occurs mainly as a part of saccharine molecule (physical incorporation of saccharin), while for $\boldsymbol{C}_{s a c}>\frac{\boldsymbol{K}_{2}-\boldsymbol{K}_{1}}{\boldsymbol{K}_{1} \cdot \boldsymbol{b}}$ the sulfur incorporates into deposit occurs as a part of metal sulfides (saccharin electroreduction mechanism).



One of the biggest challenges in the future development of electrodeposited soft high magnetic moment alloys is to further continue reducing their coercivity while simultaneously increasing their saturation magnetic flux density. The effect of saccharin concentration on resulting coercivity of $2.4 \mathrm{~T}$ CoFe alloys is shown in Figure 4, (21). As expected, the largest values of coercivity are measured for CoFe films obtained from solution without saccharin. The small additions of this additive result in significant 
decreases of the coercivity which has the lowest value for the CoFe films produced from solution containing $0.12 \mathrm{~g} \cdot \mathrm{L}^{-1}$ of saccharine. Further increasing the saccharine concentration yields $\mathrm{CoFe}$ films with higher $\mathrm{H}_{\mathrm{c}}$ values. For the $\mathrm{CoFe}$ films produced from solutions with $C_{s a c}>0.7 \mathrm{~g} \cdot \mathrm{L}^{-1}$, an insignificant difference in $H_{c}$ is observed $\left(H_{c} \approx c o n s t\right)$. This is similar to what has been reported for $\mathrm{CoNiFe}$ alloys for saccharin concentration range, $C_{s a c}>0.7 \mathrm{~g} \cdot \mathrm{L}^{-1}(16)$. A useful analysis of the results in Figure $4 \mathrm{~A}$ is achieved when the $H_{c}$ values are plotted as a function of the corresponding sulfur incorporation rates, Figure 4B. From this analysis, it is obvious that the higher incorporation rates of sulfur into magnetic deposit lead to the alloys with lower $H_{c}$ values. This data also show that in order to decrease the coercivity of the $2.4 \mathrm{~T}$ CoFe alloys produced without additives in the plating solution by $\approx 50 \%$, the incorporation rate of sulfur with addition of saccharine has to increase $\approx 6$ times. The important conclusion related to the relative contribution of each sulfur incorporation mechanism to the observed coercivity of $2.4 \mathrm{~T}$ CoFe alloys is deduced if the $H_{c}$ data in Figure 4A are plotted as a function of the ratio between the sulfur incorporation rate coming from the physical incorporation of saccharine molecules $\left(R_{M}=K_{2} \cdot \frac{b \cdot C_{s a c}}{\left(1+b \cdot C_{s a c}\right)^{2}}\right)$ and sulfur incorporation rate coming from the saccharin electroreduction, $\left(R_{E R}=K_{1} \cdot \frac{b \cdot C_{s a c}}{1+b \cdot C_{s a c}}\right)$, Figure 4C. As one can see, as long as $\mathrm{R}_{\mathrm{M}}$ represents the dominant contribution to the overall sulfur content in the $2.4 \mathrm{~T} \mathrm{CoFe}$ alloys, $R_{M} / R_{E R}>>1\left(\boldsymbol{C}_{s a c} \ll \frac{\boldsymbol{K}_{2}-\boldsymbol{K}_{1}}{\boldsymbol{K}_{1} \cdot \boldsymbol{b}}\right)$, the measured coercivity of the electrodeposited films has the lower values, between 40 and 50 Oe (Figure 4C). In the case when $R_{E R}$ makes the predominant contribution to the sulfur content in the deposit, $\mathrm{R}_{\mathrm{M}} / \mathrm{R}_{\mathrm{ER}}<<1$, $\left(\boldsymbol{C}_{\boldsymbol{s a c}}>\frac{\boldsymbol{K}_{2}-\boldsymbol{K}_{1}}{\boldsymbol{K}_{1} \cdot \boldsymbol{b}}\right.$ ) the measured coercivity of the $2.4 \mathrm{~T}$ CoFe films is significantly higher, above 60 Oe. The general conclusion that could be derived is that the most effective form of incorporated sulfur producing the softest $2.4 \mathrm{~T}$ CoFe alloys is the sulfur as a part of saccharine molecule. The incorporated sulfur as a part of the metal sulfides also contributes to the decrease in the CoFe alloy coercivity but less effectively. Therefore, the optimum design of the plating solutions for ultimately soft $2.4 \mathrm{~T}$ CoFe alloys should be such that criteria $R_{M}>>R_{E R}$ is always satisfied. 

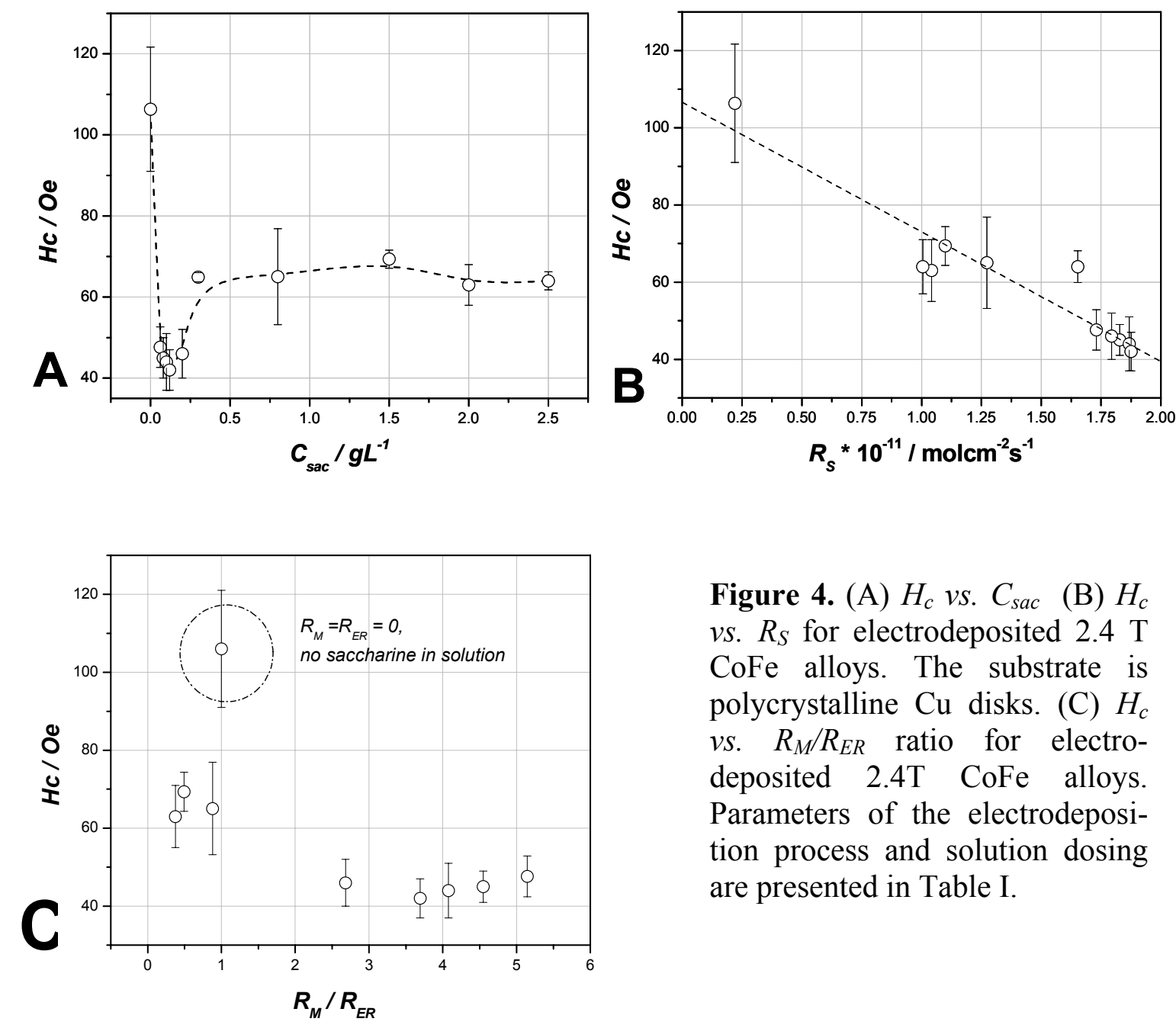

Figure 4. (A) $H_{c}$ vs. $C_{s a c}$ (B) $H_{c}$ $v s . R_{S}$ for electrodeposited $2.4 \mathrm{~T}$ CoFe alloys. The substrate is polycrystalline $\mathrm{Cu}$ disks. (C) $H_{c}$ vs. $R_{M} / R_{E R}$ ratio for electrodeposited 2.4T CoFe alloys. Parameters of the electrodeposition process and solution dosing are presented in Table I.

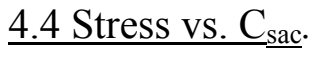

The effect of stress in the electrodeposited magnetic films has become very important phenomenon as the critical size of magnetic recoding devices and the magnetic head-media spacing in magnetic disk drives were reduced to several tens of nanometers. In general, the stress in electrodeposited soft magnetic films is dependent on many parameters. Very often, the sign of the stress in the magnetic films (compressive (-) or tensile $(+)$ ) is dependent on the final thickness of electrodeposited film, crystal structure, substrate and the film texture (26). For CoFe alloys the great part of their effective magnetic anisotropy energy is coming form the contribution of the magnetoelastic energy. The additives effectively reduce the residual stresses in the deposit and thus contribute to the overall softer properties of the CoFe films.

In Figure 5, the XRD data (2 $\theta$ peak positions) for electrodeposited CoFe films from solution having different concentration of saccharine are shown. The data deduced from $\mathrm{BCC}<110>2 \theta$ peak position shift, suggest a complex dependence of coherent strain in 
CoFe films vs. $C_{s a c}$. The coherent strain in the electrodeposited films relaxes from initially positive value (tension) towards zero and continues to build up as compressive strain reaching its maximum for, $C_{s a c} \approx 0.3 \mathrm{~g} \cdot \mathrm{L}^{-1}$. For $C_{s a c}>0.3 \mathrm{~g} \cdot \mathrm{L}^{-1}$ the coherent strain starts to relax again and eventually saturates for $C_{s a c}>1.5 \mathrm{~g} \cdot \mathrm{L}^{-1}$ as tensile strain. The correlation between the strain $v s$. $C_{s a c}$ behavior at this point is not straightforward, however the interesting observation is that coherent strain in CoFe films is close to zero (bulk alloy) for $C_{s a c} \approx 0.12 \mathrm{~g} \cdot \mathrm{L}^{-1}$ and $C_{s a c} \approx 0.7 \mathrm{~g} \cdot \mathrm{L}^{-1}$. These are the saccharine concentrations for which the maximum incorporation rate of sulfur is observed and for which our model predicts an equal contribution of the sulfur in the deposit by each of saccharine related sulfur incorporation mechanisms. More work is necessary to make the phenomenological description of this observation, yet the general conclusion is that the softest $\mathrm{CoFe}$ alloys are the ones that have the least amount of coherent strain and maximum amount of incorporated sulfur coming form the saccharine molecules.



Figure 5. $\mathrm{BCC}<110>2 \theta$ peak position as a function $C_{\text {sac }}$ for electrodeposited 2.4 $\mathrm{T}$ CoFe alloys. Solution and process details are shown in Table I.

\section{Conclusion}

The electrodeposition of soft, high magnetic moment alloys has been widely used as one of the key fabrication processes in manufacturing of magnetic recording heads for almost three decades. The desire for high magnetic moment electrodeposited materials and the need for delivering magnetic structures with dimension in the range of several tens of nanometers are the new challenges that will have to be addressed in the future fabrication of high density recording devices. This complex challenge could be addressed successfully by fully considering the conditions at the electrochemical interface for additive adsorption and incorporation, iron hydroxide formation and incorporation, and 
by careful design of the bath chemistries and processes that would yield the high moment alloys with the optimum magnetic properties.

\section{Acknowledgment}

The authors would like to thank Dr. James Meen from Texas Center for Superconductivity for help during WDS measurements. This work is sponsored by NSF Division of Electrical, Communications and Cyber Systems under the contract \# 0824215.

\section{References}

1. S. Khizroev and D. Litvinov, Perpendicular Magnetic Recording, Springer - Kluwer Academic Publishers, New York (2004).

2 . E. I. Cooper, C. Bonhote, J. Heidmann, Y. Hsu, P. Kern, J. W. Lam, M.

Ramasubramanian, N. Robertson, L. T. Romankiw and H. Xu, IBM J. Res. \& Dev., 49, 103 (2005).

3. T. Osaka, T. Yokoshima, D. Shiga, K. Imai and K. Takashima, Electrochem. Solid State Lett., 6, C53 (2003).

4. D. R. Lide, CRC Handbook of Chemistry and Physics, 86th ed., p. 8, (2005)

5 . S. R. Brankovic, X. M. Yang, T. J. Klemmer and M. Seigler, IEEE Trans. Magn., 42, 132 (2006).

6. S. R. Brankovic, S. -E. Bae and D. Litvinov, Electrochim. Acta, 53, 5934 (2008).

7 . S. R. Brankovic, K. Sendur, T. Klemmer, X. Yang and E. C. Johns, in Magnetic Material, Processes and Devices VII and Electrodeposition of Alloys, 202nd ECS Meeting Proceeding, S. Krongelb, L. T. Romankiw, Kimamoto, Chang, Judy, C. Bonhote, G. Zangari and W. Schwarzacher, Ed., p. 269, Pennington, NJ, PV 200227 (2003).

8 . S. R. Brankovic, N. Vasiljevic, T. J. Klemmer and E. C. Johns, J. Electrochem. Soc., 152, C196 (2005).

9 . T. R. Rosebrugh and W. L. Miller, J. Phys. Chem., 14, 816 (1910).

10. C. Wagner, J. Electrochem. Soc., 98, 116 (1951).

11. L. T. Romankiw and D. A. Thompson, US Patent 4, 295,173 (1981).

12 . N. Robertson, H. L. Hu and C. Tsang, IEEE Trans. Magn., 33, 2818 (1997).

13 . T. Osaka, M. Takai, K. Hayashi, K. Ohashi, M. Saito and K. Yamada, Nature, 392, 796 (1998).

14. T. Osaka, T. Yokoshima, D. Shiga, K. Imai and K. Takashima, Electrochem. SolidState Lett., 6, C53 (2003).

15. M. Schlesinger and M. Paunovic, Modern Electroplating, John Willey \& Sons, NY (2000).

16. T. Osaka, T. Sawaguchi, F. Mizutani, T. Yokoshima, M. Takai and Y. Okinaka, J. Electrochem. Soc., 146, 3295 (1999).

17 . B. N. Popov, K. -M. Yin and R. E. White, J. Electrochem. Soc., 140, 1321 (1993).

18. J. Edwards, Trans. Inst. Met. Finish, 39, 52 (1962). 
19. S. R. Brankovic, F. Wiatrowski and M. Siegler, 208 ECS Meeting Abstract, 473 Quebec City (2005)

20 . S. R. Brankovic, R. Haisimaier and N. Vasiljevic, Electrochem. Solid-State Lett., 10, D67 (2007).

21 . J George, S.-E. Bae, D. Litvinov, J. Rancheler, and S. R. Brankovic, J. Electrochemical Society, 155 D589 (2008)

22 . G. S. Frankel, V. Brusic, R. G. Schad and J. W. Chang, Corr. Sci., 35, 63 (1993).

23 . T. Osaka, M. Takai, Y. Sogawa, T. Momma, K. Ohashi, M. Saito and K. Yamada, J. Electrochem. Soc., 146, 2092 (1999).

24 . H. Kwon and A. A. Gewirth, J. Electrochem. Soc., 154, D577 (2007).

25 . C. Buessherman, Prog. Sur. Sci., 46, 335 (1994).

26 . L. B. Freund and S. Suresh, Thin Film Materials: Stress, Defect Formation, and

Surface Evolution, Cambridge University Press, (2003). 